\title{
SARS COV-2 and Pregnancy: Autoimmune Implications
}

\author{
Maria José Durango de la Ossa ${ }^{1 *}$, Maria Carolina Causil Galvis ${ }^{1}$, Andrés Iván Ramirez \\ Montes $^{1}$, John Lorenzo Delgado Lopez ${ }^{1}$, César Augusto Arroyo Pérez ${ }^{2}$, Cristian Augusto Porto \\ Hernández ${ }^{1}$ and Yamith de Jesús Álvarez Castro ${ }^{1}$ \\ ${ }^{1}$ General Physician, Universidad de Sucre, Sincelejo, Colombia \\ ${ }^{2}$ General Physician, Universidad del Magdalena, Santa Marta, Colombia
}

*Corresponding author: John Lorenzo Delgado Lopez, General medical of the University of Magdalena, Colombia

To Cite This Article: Maria José Durango de la Ossa, Maria Carolina Causil Galvis, Andrés Iván Ramirez Montes, John Lorenzo Delgado Lopez, César Augusto Arroyo Pérez, et al., SARS COV-2 and Pregnancy: Autoimmune Implications. Am J Biomed Sci \& Res. 2021 - 14(2). AJBSR. MS.ID.001964. DOI: 10.34297/AJBSR.2021.14.001964.

Received: 觜 August 30, 2021; Published: 畊 September 13, 2021

\section{Short Communication}

Historically pregnant women have been more severely affected by outbreaks of respiratory infections compared to women of similar non-pregnant age groups. This has been described in the 1918 influenza epidemic, the 1957-1958 Asian influenza epidemic, and more recently, in the $2009 \mathrm{H} 1 \mathrm{~N} 1$ and SARS pandemic in 2003 (It should be remembered that SARS-CoV is also a coronavirus, and shares $85 \%$ of the genomic sequence with SARS-CoV-2). In all these epidemics, pregnant women showed a high case fatality rate, increased risk of admission to intensive care units, increased risk of mechanical ventilation and other infectious complications [1].

Due to physiological changes in the immune and cardiorespiratory systems, pregnancy is often considered a condition of high susceptibility to viral infections, especially those that affect the respiratory system such as SARS-CoV-2. Diaphragmatic displacement through the gravid uterus and increased gestational weight are responsible for altered lung volumes leading to reduced total lung capacity and an inability to effectively clear pulmonary secretions [1].

The mother's immune status undergoes adaptive changes throughout this period; goes from a pro-inflammatory state in early pregnancy to benefit implantation and placentation to an anti-inflammatory state to benefit fetal growth during the second trimester (which may be protective and may be less severe in this population) and, finally, a pro-inflammatory state in preparation for labor [2].

The most recent literature indicates that Sars-Cov-2 infection is characterized by generating a significant increase in cytokines at the systemic level (mainly IL-2, IL-7, IL-10, tumor necrosis factor alpha, colony stimulating factor granulocytic), so that pregnant women who are in a pro-inflammatory state could present a more severe response to said infection, a consideration that should be taken into account especially during the I and III trimesters of pregnancy [2].

Although beliefs regarding the susceptibility of pregnant women to Sars-CoV-2 compared to the general population are discordant, few studies including small series of pregnant patients have shown that women had mild disease in $80 \%$ of patients. cases, severe illness in $15 \%$ of cases and critical illness in $5 \%$ of cases; According to a recent meta-analysis of 108 cases, the ICU admission rate in pregnant women was approximately $3 \%$ and the incidence of severe disease requiring mechanical ventilation was approximately $2 \%$ [3].

In conclusion, pregnant women do not appear to be more susceptible to the consequences of Sars-Cov-2 infection compared to the general population; however, it is known that physiological changes predispose pregnant women to respiratory symptoms 
during pregnancy. The foregoing, associated with changes in immunity, may be factors that determine the evolution of the COVID-19 infection. However, it remains to be determined whether these adaptations result in increased susceptibility and / or morbidity or are, in fact, protective against COVID-19 [4].

\section{References}

1. Abarzúa Camus F (2020) COVID-19 and Pregnancy. Chilean Journal of Obstetrics and Gynecology 85(2): 110-114.
2. Herrera Pérez J C, Monterio Fonseca J, Campos Sánchez S (2020) COVID-19 and pregnancy. Synergy Medical Journal 5(9): e492.

3. Merchan Villama JA R, Cedeño Cedeño S E, Rayo Caicedo K E (2020) Covid 19, pregnancy, childbirth and breastfeeding. RECIAMUC 4(3): 5868.

4. Clavero Núñez J A (2020) COVID-19 infection in pregnancy. Assistance Care. ANALS RANM 137(03): 265-269. 